To appear in Astrophysical Journal, 2007, Vol. 657, No. 1

\title{
CO line width and the black hole - bulge relationship at high redshift
}

\author{
Xue-Bing $\mathrm{Wu}$ \\ Department of Astronomy, Peking University, Beijing 100871, China \\ wuxb@bac.pku.edu.cn
}

\begin{abstract}
Recently, it has been suggested that the CO line width ( $\mathrm{FWHM}(\mathrm{CO}))$ is a surrogate for the bulge velocity dispersion $(\sigma)$ of the host galaxies of highredshift quasars, and the black hole - bulge $\left(M_{B H}-\sigma\right)$ relation obtained with this assumption departs significantly from the $M_{B H}-\sigma$ relation in the local universe. In this study, we first present an investigation of the correlation between the $\mathrm{CO}$ line width and the bulge velocity dispersion using a sample of 33 nearby Seyfert galaxies. We find that the formula adopted in previous studies, $\sigma=\operatorname{FWHM}(\mathrm{CO}) / 2.35$, is generally not a good approximation. Using it, one may underestimate the value of bulge velocity dispersion significantly when the $\mathrm{CO}$ line is narrower than $400 \mathrm{~km} \mathrm{~s}^{-1}$. By involving the galactic inclination angle $i$ as an additional parameter, we obtain a tight correlation between the inclination-corrected $\mathrm{CO}$ line width and the bulge velocity dispersion, namely, $\mathrm{FWHM}(\mathrm{CO}) / \sin \mathrm{i}=-67.16 \pm 80.18+(3.62 \pm 0.68) \sigma$. Using this new relation, we can better estimate the bulge velocity dispersion from the $\mathrm{CO}$ line width if the galactic inclination is known. We apply this new relation to nine high-redshift quasars with $\mathrm{CO}$ line detections and find that they are consistent with the local $M_{B H}-\sigma$ relation if their inclination angles are around $15^{\circ}$. The possible smaller inclinations of the high-redshift quasars are preferred because of their relatively greater likelihood of detection, and are also consistent with their relatively smaller CO line widths compared to submillimeter galaxies (SMGs) at high redshift having a similar total amount of molecular gas. Future observations are needed to confirm these results.
\end{abstract}

Subject headings: galaxies: active - galaxies: Seyfert - quasars: emission lines radio lines: galaxies 


\section{Introduction}

The masses of supermassive black holes in nearby normal and active galaxies have been reliably derived in the last two decades by using different dynamical techniques (Kormendy \& Gebhardt 2001; Ho 1999). The relationships of the black hole mass to the bulge velocity dispersion and to the bulge mass have been established for both quiescent and active galaxies (Gebhardt et al. 2000; Ferrerase et al. 2001; Tremaine et al. 2002; Onken et al. 2004). Various theoretical models have been suggested to explain the tight black hole - bulge relations (Silk \& Rees 1998; King 2003, 2005; Wyithe \& Loeb 2003; Murray, Quataert \& Thompson 2005; Begelman \& Nath 2005). Most models and simulations predict that the $M_{B H}-\sigma$ relation may be the same at different redshifts (King 2003, 2005 Wyithe \& Loeb 2003; Begelman \& Nath 2005; Robertson et al. 2006), but some do not (Kazantzidis et al. 2005). On the observational side, testing the $M_{B H}-\sigma$ relation at high redshift is a great challenge. Although we do have some methods to estimate the black hole masses of high-redshift quasars, especially by measuring their broad emission line properties (McLure \& Jarvis 2002; Vestergaard 2002; Kong et al. 2005), it is difficult to measure the bulge properties in the host galaxies of these quasars directly.

Even for quasars at low redshift, it is still not easy to resolve their host galaxies observationally because their central nuclei are too bright. Measuring the velocity dispersion of their host galaxies with spectroscopic methods is also difficult. Only very recently were the nearinfrared measurements of the bulge velocity dispersions obtained for 11 bright Palomar-Green (PG) quasars (Dasyra et al. 2006). However, for local quasars, one can use the empirical relation between the [OIII] line width and bulge velocity dispersion, $\sigma=\mathrm{FWHM}([\mathrm{OIII}]) / 2.35$, derived from local Seyfert galaxies (Nelson \& Whittle 1996), to estimate their bulge velocity dispersions and then study the $M_{B H}-\sigma$ relation for local active galactic nuclei (AGNs; Shields et al. 2003). But such an approach can not be applied to quasars at high redshift, because their [OIII] lines move out of the optical windows and are not observable.

Recently, Shields et al. (2006) suggested using the CO line width as a surrogate for the bulge velocity dispersion. CO lines have been clearly detected in about 10 quasars with redshift from 1 to 6.4 (Solomon \& Vanden Bout 2005). Their CO line widths are around several hundred $\mathrm{km} \mathrm{s}^{-1}$, comparable to the typical width of narrow emission lines of AGNs. By adopting the formula $\sigma=\mathrm{FWHM}(\mathrm{CO}) / 2.35$, Shields et al. (2006) investigated the $M_{B H}-\sigma$ relation in high-redshift quasars and found that it departs significantl from

the local $M_{B H}-\sigma$ relation. The same black hole mass may correspond to a smaller $\sigma$ value at higher redshift. However, whether or not the CO line width can be taken as a surrogate for the bulge velocity dispersion obviously deserves further study. As noted by Shields et al. (2006), the relatively narrower CO line width of high-redshift quasars may be due to their 
smaller inclinations. Face-on quasars could be preferentially found at higher redshift than quasars with larger inclinations. Clearly, more works on the CO line width and the black hole - bulge relation of high-redshift quasars are expected.

Fortunately, the CO line width and bulge velocity dispersion have been obtained for some nearby Seyfert galaxies. An early survey by Heckman et al. (1989) detected CO(1-0) emission in 18 out of 43 Seyfert galaxies using the NRAO $12 \mathrm{~m}$ telescope. With the same instrument, Maiolino et al. (1997) successfully detected $\mathrm{CO}(1-0)$ emission in 73 out of 94 Seyfert galaxies in the Revised Shapely Ames (RSA) and Center for Astrophysics (CfA) Seyfert samples (Maiolino \& Rieke 1995; Huchra \& Burg 1992). For the bulge velocity dispersion, Nelson \& Whittle (1995) have compiled a catalog of the derived values for 78 Seyfert galaxies. Therefore, we can use the Seyfert galaxies with the published data for both the CO line width and the bulge velocity dispersion to study the relationship between them. In fact, Heckman et al. (1989) have investigated such a relation in seven Seyfert galaxies (see their Table 8 and Figure 13) and found that the inclination-corrected CO line width may correlate better with the bulge velocity dispersion. Obviously, this tentative result needs to be confirmed by a larger sample of Seyfert galaxies.

In this paper, we first present an investigation of the relation between the inclinationcorrected CO line width and the bulge velocity dispersion for a relatively larger sample of Seyfert galaxies. Based on the new relation derived by us, we estimate the bulge velocity dispersions for high redshift quasars and study whether or not the $M_{B H}-\sigma$ relation at high redshift is different from the local one. Finally we give our summary and discussions.

\section{CO line width and velocity dispersion of Seyfert galaxies}

CO lines have been successfully detected in a number of Seyfert galaxies (Heckman et al. 1989; Maiolino et al. 1997; Vila-Vilaro, Taniguchi \& Nakai 1998; Curran, Aalto \& Booth 2000). To avoid the selection bias, we use the Seyfert sample in Maiolino et al. (1997), for which the sources were selected from the RSA and CfA surveys. Of 73 CO-detected Seyfert galaxies in this sample, we find 33 have measured bulge velocity dispersion data, either from the catalog of Nelson \& Whittle (1995) or from the Hyperleda database 1 (Paturel et al. 2003). We use this sample of 33 Seyfert galaxies (hereafter called the main sample) to investigate the relation between the $\mathrm{CO}$ line width and the bulge velocity dispersion for AGNs. We also find eight additional AGNs, including four Seyfert galaxies in Heckman et al. (1989) and four radio galaxies in Evans et al. (2005), both with measured data for the

\footnotetext{
${ }^{1}$ see http://leda.univ-lyon1.fr
} 
CO line width and the bulge velocity dispersion. These eight sources are not included in the statistical studies, but will be used to check our results. The data of 33 Seyfert galaxies in our main sample and eight additional sources are listed in Table 1. The galactic disk inclination angles of these AGNs can be obtained from the Hyperleda database. However, for NGC 6814 we take an inclination of $20^{\circ}$ from Whittle (1992), rather than the value $82^{\circ}$ given by Hyperleda because it seems more likely to be a face-on galaxy, according to its infrared image (Mulchaey, Regan \& Kundu 1997). For other sources, the inclination data listed in Whittle (1992) are generally consistent with those given by Hyperleda.

First, we directly investigate the relation between the $\mathrm{CO}$ line width and the bulge stellar velocity dispersion for Seyfert galaxies. In Figure 1 we plot such a relation for 33 Seyferts in our main sample and 8 additional sources. Clearly, there is a good correlation between them. For 33 Seyferts in our main sample, the Spearman correlation coefficient is 0.63 , and the null probability is $8.95 \times 10^{-5}$. Using the Ordinary Least Square (OLS) bisector method (Isobe et al. 1990), we obtain a fitted relation,

$$
\operatorname{FWHM}(\mathrm{CO})=-90.66 \pm 68.95+(2.90 \pm 0.58) \sigma .
$$

Comparing the relation $\sigma=\mathrm{FWHM}(\mathrm{CO}) / 2.35$ with the fitted line and the observational data for Seyferts, we find that, although this previously suggested relation is not a bad approximation for the correlation, by using it one can underestimate the $\sigma$ value for objects with FWHM(CO) less than $400 \mathrm{~km} \mathrm{~s}^{-1}$ and overestimate the $\sigma$ value for objects with broader CO lines.

In a previous study, Heckman et al. (1989) mentioned that the CO line width correlates well with the galactic inclination, and when the $\mathrm{CO}$ line width is corrected for inclination, its correlation with the bulge velocity dispersion can be improved. With an enlarged sample of 33 Seyferts, we can re-investigate the effect of inclination. In Fig. 2 we plot the FWHM(CO)/ $\sigma$ versus the sine of the inclination. Apparently, there is a correlation between them. The Spearman correlation coefficient is 0.60 and the null probability is $2.28 \times 10^{-4}$. The OLS bisector fit gives

$$
\mathrm{FWHM}(\mathrm{CO}) / \sigma=-0.58 \pm 0.30+(3.95 \pm 0.44) \sin \mathrm{i}
$$

From Fig. 2 we can clearly see that the relation $\sigma=\mathrm{FWHM}(\mathrm{CO}) / 2.35$ is generally not a good approximation. Using it one can significantly underestimate $\sigma$ from the CO line width for the AGNs with smaller inclination, and overestimate $\sigma$ for AGNs with higher inclination. Only for galaxies with an inclination of about $45^{\circ}(\sin i$ around 0.7$)$, does the relation $\sigma=\mathrm{FWHM}(\mathrm{CO}) / 2.35$ apply. From Fig. 2 we can also see that the galactic inclinations of both Seyfert 1 and Seyfert 2 galaxies seem to span a similar range. However, we do see that there are relatively more Seyfert 2 galaxies with inclinations larger than $70^{\circ}$ 
than Seyfert 1 galaxies (see also Table 1). In our main sample three Seyfert and one Seyfert 1 galaxy have inclinations larger than $70^{\circ}$. This Seyfert 1, NGC 2992, is actually an edge-on Seyfert 1.9 galaxy. We also note that two Seyfert 2 galaxies with the smallest inclinations in our sample, NGC 5929 and NGC 1068, are both intrinsically Seyfert 1 galaxies with polarized broad emission lines ( $\mathrm{Gu} \&$ Huang 2002). Considering the possible selection effect in our incomplete CO-detected Seyfert sample and also the complicated relation between the inclinations of a central accretion disk and host galaxy, the apparent distributions of galactic inclinations of Seyfert galaxies may not be too hard to understand.

Finally, we investigate the relation between the inclination-corrected $\mathrm{CO}$ line width $(\mathrm{FWHM}(\mathrm{CO}) / \sin i)$ and the bulge velocity dispersion. Using seven Seyfert galaxies, Heckman et al. (1989) previously obtained $\operatorname{FWHM}(\mathrm{CO}) / \sin \mathrm{i}=-20+3.57 \sigma$. In Fig. 3 we plot such a relation for 33 Seyferts in our main sample and 8 additional sources. For 33 Seyferts in our main sample, the Spearman correlation coefficient is 0.71 , and the null probability is $3.18 \times 10^{-6}$. Comparing with the correlation between $\mathrm{FWHM}(\mathrm{CO})$ and $\sigma$, the correlation is improved when the CO line width is corrected for inclination. For 33 Seyferts in our main sample, we obtain an OLS bisector fit as

$$
\mathrm{FWHM}(\mathrm{CO}) / \sin \mathrm{i}=-67.16 \pm 80.18+(3.62 \pm 0.68) \sigma .
$$

We note that this relation is in good agreement with the previous result obtained from a smaller Seyfert sample (Heckman et al. 1989). From Fig. 3 we see that the inclinationcorrected CO line width for both the Seyferts in our main sample and eight additional AGNs correlates well with the bulge velocity dispersion, with only a few outliers. The most extreme outlier in Fig. 3 is the radio galaxy 3C 84 (Perseus A), which has a narrower CO line width $\left(200 \mathrm{~km} \mathrm{~s}^{-1}\right)$ relative to its bulge velocity dispersion value $\left(272 \mathrm{~km} \mathrm{~s}^{-1}\right)$. After a close look at the $\mathrm{CO}(1-0)$ spectrum of 3C 84 in Fig. 1 of Evans et al. (2005), we note that its narrower CO line width was probably obtained by measuring the central peak profile only. There are also clear subpeaks in both the red and blue sides around the central peak. Therefore, we guess that the $\mathrm{CO}$ line width of $3 \mathrm{C} 84$ may be significantly underestimated. This obviously needs to be confirmed by more accurate observations in the future.

The reason for considering the inclination correction of the $\mathrm{CO}$ line width when investigating its relation to the bulge velocity dispersion is straightforward. As indicated by Hackman et al. (1989), unlike the [OIII] lines in the narrow-line region, CO gas is probably arrayed in a rotating disk that is coplanar with the galaxy disk. When we view the CO gas with an inclination, what we actually measure is its projected velocity. Therefore, the inclination-corrected CO line width may better represent the intrinsic CO velocity. Therefore, it is not surprising that such an intrinsic $\mathrm{CO}$ velocity has a better correlation with the stellar velocity dispersion in the central bulge than the observed CO line width. 


\section{Application to high-redshift quasars}

CO lines have been detected for some high redshift quasars (Solomon \& Vanden Bout 2005) and therefore offer us an opportunity to study the black hole - bulge relation at high redshift. Using the UV/optical broad emission line and continuum properties, Shields et al. (2006) have estimated the black hole mass of these high-redshift quasars with the methods suggested in some previous studies (Kaspi et al. 2000, 2005; Vestergaard 2002; McLure \& Dunlop 2004). Taking the CO line width as a surrogate for the bulge velocity dispersion $(\sigma=\mathrm{FWHM}(\mathrm{CO}) / 2.35)$, Shields et al. (2006) found that these high-redshift quasars evidently depart from the usual $M_{\mathrm{BH}}-\sigma$ relation in the local universe and thus suggested that the giant black holes at high redshift reside in the undersized bulge. As we know from our investigations above, the observed CO line width needs to be corrected for inclination. In this section, we use the tightest relation (Eq. 3) we found for Seyfert galaxies in the section above to re-investigate the black hole - bulge relation at high redshift.

We note that the host galaxy morphologies of local Seyfert galaxies and high-redshift quasars may not be the same. Indeed, the host galaxies of Seyfert galaxies are typically spiral, while those of high-redshift quasars are rather complex and usually disturbed. However, there also appears to be spiral structure in the central part of host galaxies of high-redshift quasars (Hutchings 2004). On the other hand, observations show that the host galaxy morphologies of Seyfert galaxies are mostly asymmetric, probably related to the star-forming activities (Maiolino et al. 1997). Similarly, the complex host galaxy morphologies of high-redshift quasars are probably also due to the triggers of AGNs and star-forming activities. The same as Seyfert galaxies, high-redshift quasars are mostly radio-quiet, and their masses of total molecular gas are also mainly in the range $10^{9} M_{\odot}-10^{11} M_{\odot}$ (Solomon \& Vanden Bout 2005). Therefore, based on these points, we tentatively assume that the CO dynamics in high-redshift quasars is not dramatically different from the local Seyfert galaxies and apply our derived relation between the inclination-corrected CO line width and bulge velocity dispersion to high-redshift quasars. Obviously, this assumption needs to be confirmed by further studies.

Shields et al. (2006) have listed the observed CO line width and the estimated black hole mass for nine high-redshift quasars with CO detection in their Table 2. As there is no information about the inclination of the host galaxy of these high-redshift quasars, it is difficult to derive the inclination-corrected CO line width for them. However, one usually believes that a quasar at high redshift should be more easily detected if its galaxy disk is face-on. Recently, Greve et al. (2005) and Carilli \& Wang (2006) have found the clear difference in the CO line widths of quasar host galaxies and SMGs at high redshift. The CO line widths of high-redshift quasars are typically a factor of 2.3 narrower than those of 
other SMGs with the same amount of molecular gas. Carilli \& Wang (2006) argued that the difference in the CO line width distribution of high-redshift quasars and SMGs can be explained if the average inclination of quasars is about 3 times smaller than that of SMGs.

Assuming that the average galaxy inclination of nine high-redshift quasars with $\mathrm{CO}$ detections is $15^{\circ}$, we can use Eq. (3) to derive the bulge velocity dispersion from the $\mathrm{CO}$ line width and then investigate the $M_{\mathrm{BH}}-\sigma$ relation for high-redshift quasars. Our result is shown in Fig. 4, from which we can clearly see that if we use the inclination-corrected CO line width to estimate the bulge velocity dispersion, the $M_{\mathrm{BH}}-\sigma$ relation for high-redshift quasars is generally consistent with the local one. In fact, we find that the data point for each quasar can match the local $M_{\mathrm{BH}}-\sigma$ relation very well if we assume the inclination in the range $10^{\circ}-30^{\circ}$. Obviously, our result is different from that recently obtained by Shields et al. (2006), who used $\sigma=\mathrm{FWHM}(\mathrm{CO}) / 2.35$ to estimate the bulge velocity dispersions for high-redshift quasars. Therefore, we think it is still premature to claim that the black hole bulge relationship at high redshift is different from the local one. The giant black hole masses of these high-redshift quasars relative to their narrower $\mathrm{CO}$ line width can be explained by assuming smaller inclinations. If the inclinations of these quasars are really smaller, the bulge velocity dispersion values derived from the inclination-corrected $\mathrm{CO}$ line widths will be in the range $250 \mathrm{~km} \mathrm{~s}^{-1}-500 \mathrm{~km} \mathrm{~s}^{-1}$, making them consistent with the local $M_{\mathrm{BH}}-\sigma$ relation.

Shields et al. (2006) mention that most of the low-redshift PG quasars seem to follow the usual $M_{\mathrm{BH}}-\sigma$ relation, even when using $\sigma=\mathrm{FWHM}(\mathrm{CO}) / 2.35$. This can be understood if these quasars have inclinations in a range between $30^{\circ}$ and $45^{\circ}$. From Fig. 2 and Eq. (3), we can see that only if the inclinations are in such a range is the formula $\sigma=\mathrm{FWHM}(\mathrm{CO}) / 2.35$ not a bad approximation, so the $\sigma$ value derived with this formula may not deviate too much from the value derived by using Eq. (3). However, we noted that even in the low-redshift PG quasar sample there are also several narrow CO quasars, with CO line width in the range $50 \mathrm{~km} \mathrm{~s}^{-1}-90 \mathrm{~km} \mathrm{~s}^{-1}$ (Evans et al. 2001, 2006; Shields et al. 2006). These objects also seem to be outliers of the $M_{\mathrm{BH}}-\sigma$ relation (see Fig. 1 of Shields et al. 2006). If we assume the smaller inclinations $\left(10^{\circ}-15^{\circ}\right)$ for these narrow CO quasars, by deriving the inclination-corrected $\sigma$ value, we can see that they can also fit the $M_{\mathrm{BH}}-\sigma$ relation. Shields et al. (2006) suspect that these narrow CO quasars are starbursts confined to a small part of their host galaxies. However, the possibility of smaller inclinations for these quasars can not be excluded.

In the above investigations we adopted the black hole masses for quasars given by Shields et al. (2006), who assumed random orbits of the broad-line region (BLR) clouds. In fact, if the BLR is also in a disk like configuration, the BLR inclinations can also affect the black hole 
mass estimations (McLure \& Dunlop 2001; Wu \& Han 2001; Collin et al. 2006). If we assume a BLR inclination angle $i$ and a ratio $A$ of the random velocity component to the planar velocity component, the black hole mass increases by a factor of $1 / 3\left(\sin ^{2} i+A^{2}\right)$ (see Eq. (4) in $\mathrm{Wu} \&$ Han 2001), compared with that obtained by assuming a random orbit of BLR as done in Shields et al. (2006). If we assume that the BLR inclinations of high-redshift quasars are around $15^{\circ}$ and $A$ is in the range $0-0.3$ (Collin et al. 2006), their black hole masses are larger by a factor of 2 - 5 than the masses given by Shields et al. (2006). This means that the $M_{B H}-\sigma$ relation for the high-redshift quasars would be more offset from the local one if the CO line width was not corrected for inclination. However, if we assume that the BLR inclination is about the same as the galactic inclination for high-redshift quasars and consider the inclination effects on both the black hole mass and bulge velocity dispersion estimations, we find that the $M_{B H}-\sigma$ relation for high-redshift quasars can still be consistent with the local one if the inclination angles are around $10^{\circ}$. Our conclusion is almost unchanged, even if the inclination effect on the black hole mass is considered. because $M_{B H} \propto \sigma^{4}$, which indicates that the inclination dependence of $\sigma^{4}\left(\propto 1 / \sin ^{4} i\right)$ is much stronger than that of $M_{B H}\left(\propto 1 / \sin ^{2} i\right)$. For the low-redshift PG quasars, if their inclinations are around $30^{\circ}-$ $45^{\circ}$, the inclination will have less effect on their $M_{B H}-\sigma$ relation, because the inclination corrections to both $M_{B H}$ and $\sigma$ are much smaller. Among 11 PG quasars with stellar velocity dispersions recently measured by CO absorption features (Dasyra et al. 2006), seven have detected CO emissions (Evans et al. 2001; Scoville et al. 2003; see also Shields et al. 2006). Using the $\sigma$ and FWHM of CO emission-line data for these seven quasars, from Eq. (3) we can roughly estimate their inclination values to be in the range $20^{\circ}-45^{\circ}$, consistent with that we assumed for PG quasars. However, we must note that the BLR geometry and dynamics may not be as simple as we assumed. More accurate determinations of black hole masses, especially for high-redshift quasars, are still needed.

\section{Summary and discussions}

We have presented an investigation of the correlation between the CO line width and the bulge velocity dispersion using a sample of 33 nearby Seyfert galaxies. We found that the formula adopted in some previous studies, $\sigma=\mathrm{FWHM}(\mathrm{CO}) / 2.35$, is generally not a good approximation. Using it, one may significantly underestimate the value of velocity dispersion when the CO line is narrower than $400 \mathrm{~km} \mathrm{~s}^{-1}$. By involving the galactic inclination angle as an additional parameter, we obtained a tight relation between the inclination-corrected $\mathrm{CO}$ line width $\mathrm{FWHM}(\mathrm{CO}) / \sin i$ and $\sigma$ (see Eq. (3)). Using this new relation, we can better estimate the bulge velocity dispersion from the CO line width. We applied this new relation to nine high-redshift quasars with $\mathrm{CO}$ line detections and found that they are consistent 
with the local $M_{B H}-\sigma$ relation if their inclination angles are around $15^{\circ}$. Therefore, we think that it is premature to conclude that the high-redshift quasars do not follow the local $M_{B H}-\sigma$ relation based on a simple $\sigma-\mathrm{FWHM}(\mathrm{CO})$ relation without considering the effects of galactic inclinations.

There are three important assumptions in our study. One is that we assume that the $\mathrm{CO}$ emissions are not uniform and are mainly concentrated in a rotating galactic disk. This is probably true because the molecular gas has been seen in such a configuration at subkiloparsec scales in most low-redshift ultraluminous infrared galaxies (Downes \& Soloman 1998). In addition, the double-peaked CO line profiles have been frequently seen in Seyfert galaxies, low-redshift quasars and SMGs (Maiolino et al. 1997; Evans et al. 2001; Greve et al. 2005). These profiles are likely to be produced if the molecular gas is in a rotating disk, although such an explanation is not unique (Carilli \& Wang 2006). The second assumption we have made is the smaller inclinations of the high-redshift quasars. The smaller inclinations (that is, a face-on geometry) of these high-redshift quasars are indeed preferred because we would be unable to detect them if they were viewed edge-on. Moreover, the smaller inclinations of these high-redshift quasars are also consistent with their relatively smaller CO line widths in comparison with other SMGs at high redshift having similar total amount of molecular gas (Greve et al. 2005; Carilli \& Wang 2006), although we can not exclude other possibilities for the narrowness of CO lines of these quasars, such as the different stages of the merge sequence or galaxy mass or size (see discussions in Carilli \& Wang 2006). The third assumption is that the CO dynamics for the local Seyfert galaxies and high-redshift quasars are not dramatically different. This obviously needs to be confirmed by future studies. The future Atacama Large Millimeter Array (ALMA) telescope will have both high sensitivity and resolution, and will be able to easily reveal the $\mathrm{CO}$ dynamics of high-redshift quasars.

In order to get a better understanding of the $M_{B H}-\sigma$ relation at high redshift, we have to rely on more accurate measurements of both $M_{B H}$ and $\sigma$ for high-redshift quasars and galaxies in the future. The CO imaging and spectroscopic studies on these distant objects with more advanced future instruments will tell us more about the geometry and dynamics of the molecular gas, as well as their relation to the galactic bulge. These studies are absolutely needed to check the relationship between the CO line width and the bulge velocity dispersion we derived here. In addition, much work is needed to do to get better estimates of the black hole masses for distant quasars using various more advanced techniques. These efforts will undoubtedly greatly improve our knowledge about the black hole - bulge relation in the early universe.

I thank Chris Carilli, Yu Gao, Fukun Liu, Ran Wang and Bingxiao Xu for stimulating discussions and the anonymous referee for helpful suggestions. This work is supported by 
NSFC grants (10473001 and 10525313), an RFDP grant (20050001026), and the Key Grant

Project of the Chinese Ministry of Education (305001). I acknowledge use of the HyperLeda database (http://leda.univ-lyon1.fr).

\section{REFERENCES}

Carilli, C.L. \& Wang, R. 2006, AJ, 131, 2763

Collin, S., Kawaguchi, T., Peterson, B. M., Vestergaard, M. 2006, A\&A, 456, 75

Curran, S. J., Aalto, S., Booth, R. S. 2000, A\&AS, 141, 193

Dasyra et al. 2006, ApJ, 656, in press (astro-ph/0610719)

Downes, D. \& Soloman, P. 1998, ApJ, 507, 615

Evans, A.S., Frayer, D. T., Surace, J. A. \& Sanders, D. B. 2001, AJ, 121, 3285

Evans, A.S., Mazzarella, J.M., Surace, J.A., Frayer, D.T., Iwasawa, K., Sanders, D.B. 2005, ApJS, 159, 197

Evans, A.S., Soloman, P. M., Tacconi, L. J., Vavilkin, T., Downes, D. 2006, AJ, in press

Ferrarese, L., et al. 2001, ApJ, 555, L79

Gebhardt, K. et al. 2000, ApJ, 539, L13

Greve, T. et al. 2005, MNRAS, 359, 1165

Gu, Q. \& Huang, J. 2002, ApJ, 579, 205

Heckmann, T.M., Blitz, L., Wilson, A.S., Armus, L. 1989, ApJ, 342, 735

Heckmann, T.M., Illingworth, G.D., Miley, G.R., van Breugel, W.J.M. 1985, ApJ 299, 41

Heraudean, Ph., Simien, F. 1998, A\&AS 133, 317

Ho, L.C. 1999, in Observational Evidence for Black Holes in the Universe, ed. S.K. Charkrabarti (Dordrecht: Kluwer), 157

Huchra, J. \& Burg, R. 1992, ApJ, 393, 90

Hutchings, J. B. 2004, in Proceedings of IAU Symposium, No. 222. Eds. by T. StorchiBergmann, L.C. Ho, and Henrique R. Schmitt. Cambridge University Press, 523 
Isobe, T., Feigelson, E. D., Akritas, M. G., \& Babu, G. J. 1990, ApJ, 364, 104

James, P., Bate, C., Wells, M., Wright, G., Doyon, R. 1999, MNRAS, 309, 585

Kaspi, S., Maoz, D., Netzer, H., Peterson, B. M., Vestergaard, M., \& Jannuzi, B. T. 2005, ApJ, 629, 61

Kaspi, S., Smith, P. S., Netzer, H., Maoz, D., Jannuzi, B. T., \& Giveon, U. 2000, ApJ, 533, 631

Kazantzidis et al. 2005, ApJ, 623, 67

King, A. R. 2003, ApJ, 596, L27

King, A. R. 2005, ApJ, 635, L121

Kong, M.-Z., Wu, X.-B., Wang, R., Han, J.-L. 2006, ChJAA, 6, 396

Kormendy, J., \& Gebhardt, K. 2001, in AIP conference proceedings, Vol. 586. Edited by J. Craig Wheeler and Hugo Martel. ISBN 0735400261, p.363

Longo, G., Busarello, G., Lorenz, H., Richter, G., Zagglia, S. 1994, A\&A 282, 418

Maiolino, R., \& Rieke, G. H. 1995, ApJ, 454, 95

Maiolino, R., Ruiz, M., Rieke, G. H., Papadopoulos, P. 1997, ApJ, 485, 552

McLure, R.J., \& Dunlop, J. S. 2001, MNRAS, 327, 199

McLure, R.J., \& Dunlop, J. S. 2004, MNRAS, 352, 1390

McLure, R.J., Jarvis, M.J. 2002, MNRAS, 337, 109

Mulchaey, J. S., Regan, M., \& Kundu A. 1997, ApJS, 110, 299

Murray, N. Quataert, E., \& Thompson, T. A. 2005, ApJ, 490, 493

Nelson, C.H., \& Whittle, M. 1995, ApJS, 99, 67

Nelson, C.H., \& Whittle, M. 1996, ApJ, 465, 96

Oliva, E., Origlia, L., Kotilainen, J.K., Moorwood, A.F.M. 1995, A\&A 301, 55

Oliva, E., Origlia, L., Maiolino, R., Moorwood, A. F. M. 1999 A\&A 350, 9 
Onken, C. A., Ferrarese, L., Merritt, D., Peterson, B. M., Pogge, R. W., Vestergaard, M., Wandel, A. 2004, ApJ, 615, 645

Paturel G., Petit, C., Prugniel, Ph., Theureau, G., Rousseau, J., Brouty, M., Dubois, P., Cambresy, L. 2003, A\&A, 412, 45

Robertson, B. et al. 2006, ApJ, 641, 90

Scoville, N. Z., Frayer, D. T., Schinnerer, E., Christopher, M. 2003, ApJ, 585, L105

Shaw, M., Wilkinson, A., Carter, D. 1993, A\&A 268, 511

Shields, G. A., Gebhardt, K., Salviander, S., Wills, B. J., Xie, B., Brotherton, M. S., Yuan, J., Dietrich, M. 2003, ApJ, 583, 124

Shields, G. A., Menezes, K. L., Massart, C. A., Vanden Bout, P. 2006, ApJ, 641, 683

Shier, L.M., Fisher, J. 1998 ApJ 497, 163

Silk, J., Rees, M. J. 1998, A\&A, 331, L1

Smith, E.P., Heckman, T.M., Illingworth, G.D. 1990 ApJ 356, 399

Solomon, P. \& Vanden Bout, P. 2005, ARA\&A, 43, 677

Terlevich, E., Diaz, A. I., Terlevich, R. 1990, MNRAS, 242, 271

Tremaine, S. et al. 2002, ApJ, 574, 740

Vestergaard, M. 2002, ApJ, 571, 733

Vila-Vilaro, B., Taniguchi, Y., Nakai, N. 1998, AJ, 116, 1553

Whittle, M. 1992, ApJS, 79, 49

Wu, X.-B. \& Han, J. L. 2001, ApJ, 561, L59

Wyithe, J. S. B., \& Loeb, A. 2003, ApJ, 595, 614 


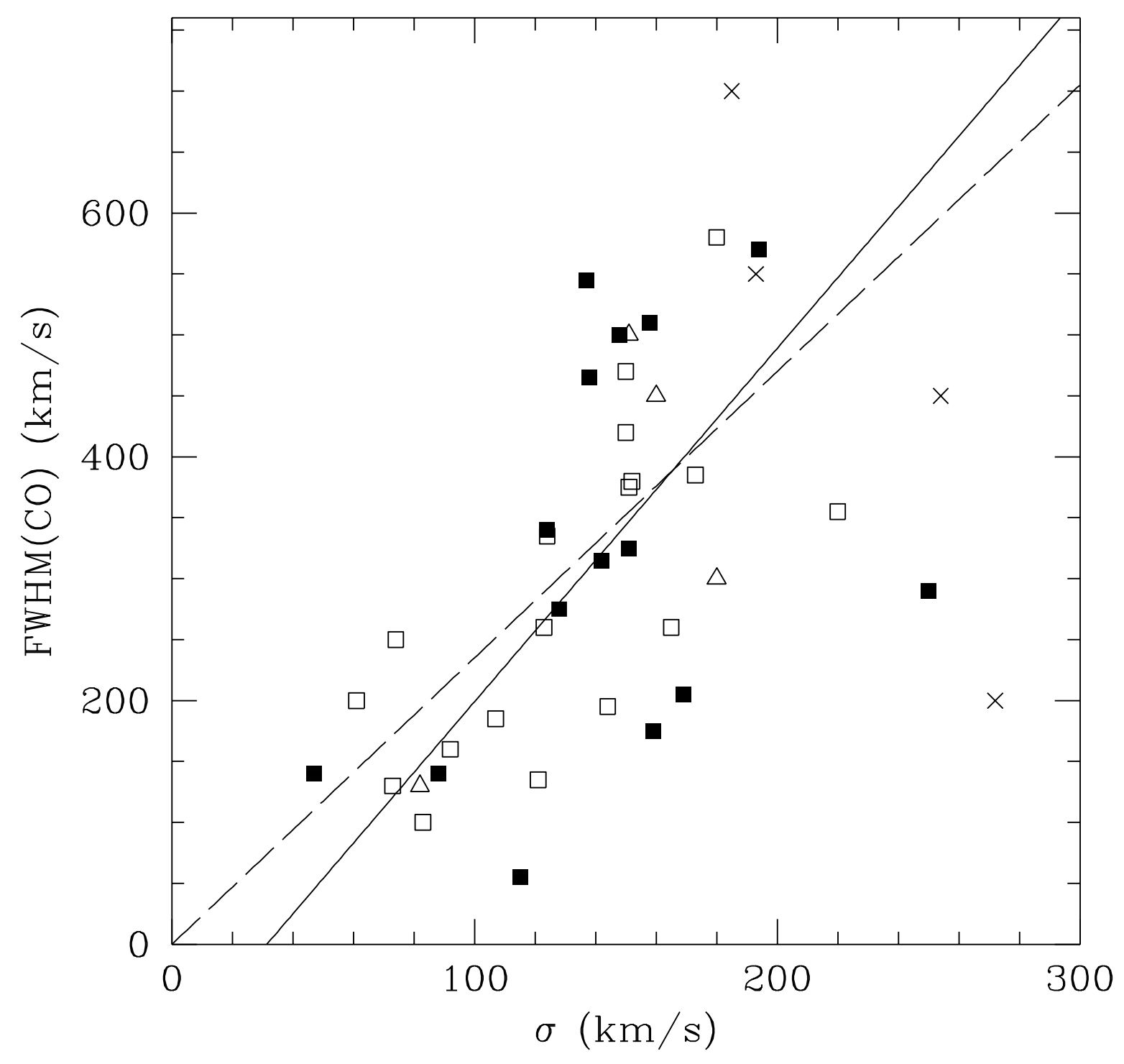

Fig. 1. - The relation between the CO line width and the bulge stellar velocity dispersion. The filled and open squares represent Seyfert 1s and Seyfert 2s in our main sample. The triangles represent 4 additional Seyferts in Heckman et al. (1989) and the crosses represent 4 radio galaxies in Evans et al. (2005). The solid line represents the OLS besector fit. The dashed line shows $\sigma=\mathrm{FWHM}(\mathrm{CO}) / 2.35$. 


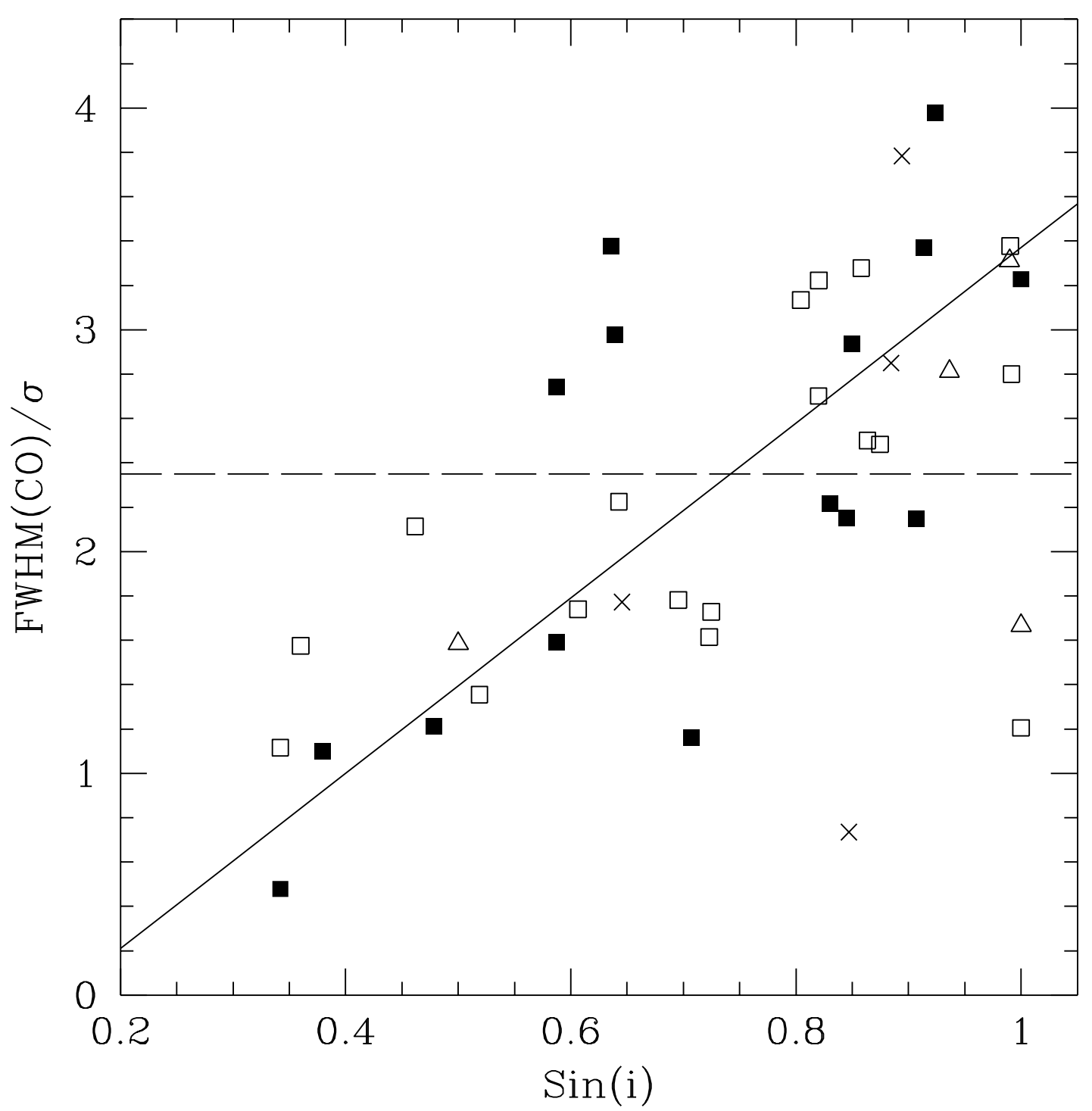

Fig. 2.- The relation of the ratio of $\mathrm{CO}$ line width and the bulge velocity dispersion with the galactic inclination. The solid line shows the OLS bisector fit. The dashed line shows $\sigma=\mathrm{FWHM}(\mathrm{CO}) / 2.35$. The symbols have the same meanings as in Fig. 1. 


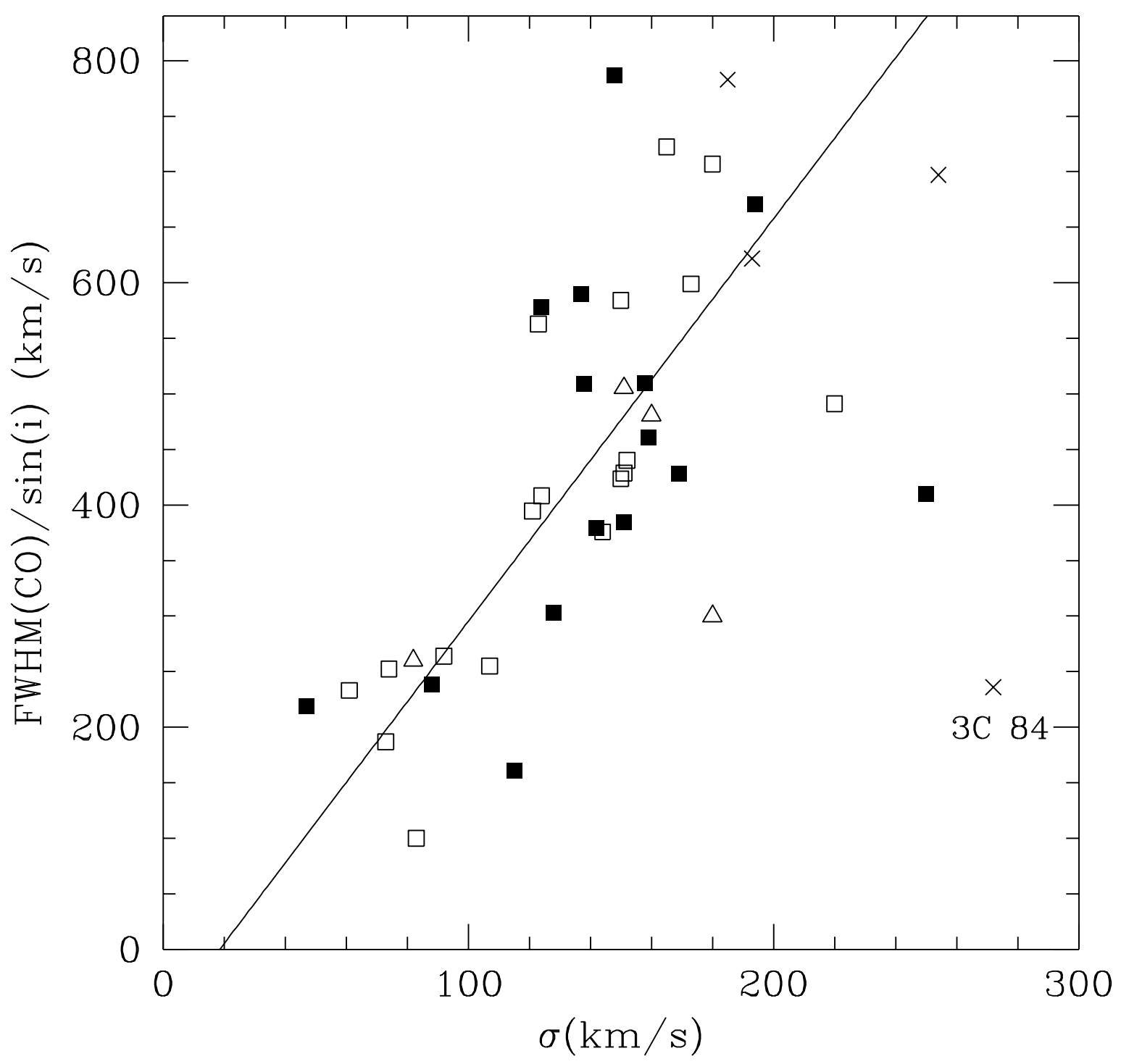

Fig. 3.- The relation between the inclination-corrected $\mathrm{CO}$ line width and the bulge velocity dispersion. The solid line shows the OLS bisector fit. The symbols have the same meanings as in Fig. 1. The extreme outlier object 3C 84 is indicated. 


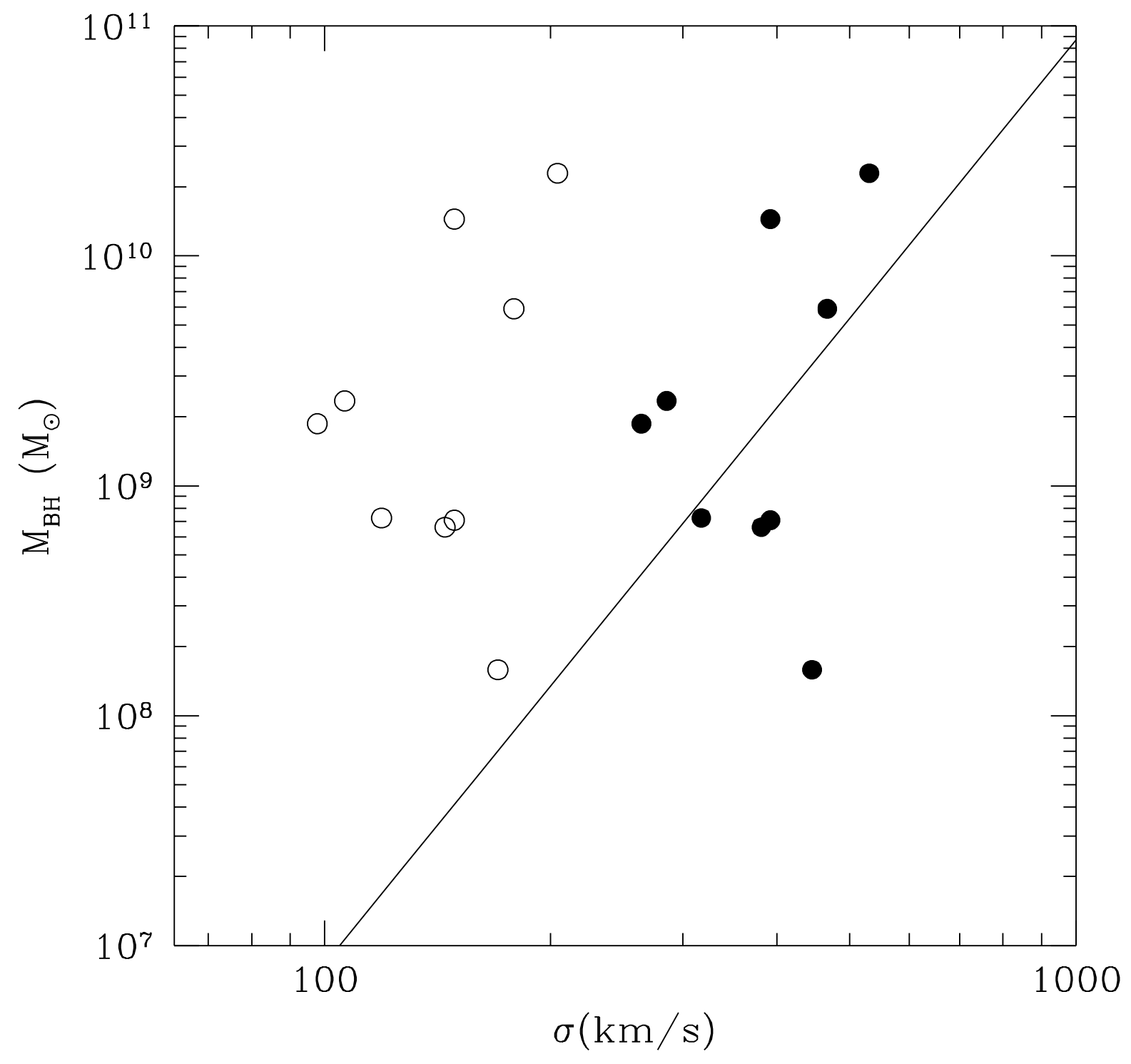

Fig. 4. - The $M_{\mathrm{BH}}-\sigma$ relation for high redshift quasars. The open circles correspond to $\sigma$ values derived from $\sigma=\mathrm{FWHM}(\mathrm{CO}) / 2.35$, while the filled circles correspond to $\sigma$ values derived from the inclination-corrected $\mathrm{CO}$ line width by assuming the inclination of $15^{\circ}$. The solid line shows the local $M_{\mathrm{BH}}-\sigma$ relation given by Tramaine et al. (2002). 
Table 1. The data of 33 Seyferts in our main sample and 8 additional AGNs

\begin{tabular}{|c|c|c|c|c|c|c|c|}
\hline Name & Type $^{a}$ & $\mathrm{z}$ & $\begin{array}{l}\mathrm{FWHM}(\mathrm{CO}) \\
\quad\left(k m s^{-1}\right)\end{array}$ & $\operatorname{Ref}^{b}$ & $\begin{array}{c}\sigma \\
\left(k m s^{-1}\right)\end{array}$ & $\operatorname{Ref}^{c}$ & $\begin{array}{c}\mathrm{i}^{d} \\
\text { (degree) }\end{array}$ \\
\hline NGC 513 & $\mathrm{~S} 2$ & 0.0195 & 380 & M97 & $152 \pm 10$ & NW95 & 59.7 \\
\hline Mrk 573 & $\mathrm{~S} 2$ & 0.0169 & 260 & M97 & $123 \pm 16$ & NW95 & 27.5 \\
\hline NGC 863 & $\mathrm{~S} 1$ & 0.0263 & 205 & M97 & $169 \pm 28$ & NW95 & 28.6 \\
\hline NGC 1068 & $\mathrm{~S} 2$ & 0.0038 & 260 & M97 & $165 \pm 12$ & NW95 & 21.1 \\
\hline NGC 1365 & $\mathrm{~S} 1$ & 0.0054 & 325 & M97 & $151 \pm 20$ & OOKM95 & 57.7 \\
\hline NGC 1667 & $\mathrm{~S} 2$ & 0.0151 & 385 & M97 & $173 \pm 17$ & TDT & 40.0 \\
\hline NGC 2110 & $\mathrm{~S} 2$ & 0.0076 & 355 & M97 & $220 \pm 25$ & NW95 & 46.3 \\
\hline NGC 2273 & $\mathrm{~S} 2$ & 0.0062 & 335 & M97 & $124 \pm 10$ & NW95 & 55.1 \\
\hline Mrk 10 & $\mathrm{~S} 1$ & 0.0292 & 545 & M97 & $137 \pm 38$ & NW95 & 67.5 \\
\hline NGC 2992 & $\mathrm{~S} 1$ & 0.0077 & 510 & M97 & $158 \pm 13$ & NW95 & 90.0 \\
\hline Mrk 1239 & $\mathrm{~S} 1$ & 0.0196 & 290 & M97 & $250 \pm 20$ & OOMM99 & 45.0 \\
\hline NGC 3079 & $\mathrm{~S} 2$ & 0.0037 & 420 & M97 & $150 \pm 10$ & SWC93 & 82.5 \\
\hline NGC 3185 & $\mathrm{~S} 2$ & 0.0041 & 200 & M97 & $61 \pm 20$ & NW95 & 59.1 \\
\hline NGC 3227 & $\mathrm{~S} 1$ & 0.0038 & 275 & M97 & $128 \pm 13$ & NW95 & 65.1 \\
\hline NGC 3362 & $\mathrm{~S} 2$ & 0.0277 & 160 & M97 & $92 \pm 28$ & NW95 & 37.3 \\
\hline NGC 3786 & $\mathrm{~S} 1$ & 0.0090 & 315 & M97 & $142 \pm 13$ & NW95 & 56.1 \\
\hline NGC 4051 & $\mathrm{~S} 1$ & 0.0023 & 140 & M97 & $88 \pm 13$ & NW95 & 36.0 \\
\hline NGC 4388 & $\mathrm{~S} 2$ & 0.0084 & 250 & M97 & $74 \pm 34$ & NW95 & 82 \\
\hline NGC 4501 & $\mathrm{~S} 2$ & 0.0076 & 375 & M97 & $151 \pm 17$ & HS98 & 61 \\
\hline NGC 4593 & $\mathrm{~S} 1$ & 0.0083 & 340 & M97 & $124 \pm 29$ & NW95 & 36.0 \\
\hline NGC 5033 & $\mathrm{~S} 1$ & 0.0029 & 465 & M97 & $138 \pm 10$ & HS98 & 66.0 \\
\hline NGC 5347 & $\mathrm{~S} 2$ & 0.0079 & 130 & M97 & $73 \pm 14$ & NW95 & 44.1 \\
\hline NGC 5548 & $\mathrm{~S} 1$ & 0.0171 & 140 & M97 & $47 \pm 67$ & NW95 & 39.7 \\
\hline NGC 5929 & $\mathrm{~S} 2$ & 0.0085 & 135 & M97 & $121 \pm 13$ & NW95 & 20.0 \\
\hline NGC 5940 & $\mathrm{~S} 1$ & 0.0340 & 175 & M97 & $159 \pm 49$ & NW95 & 22.3 \\
\hline NGC 5953 & $\mathrm{~S} 2$ & 0.0065 & 185 & M97 & $107 \pm 8$ & NW95 & 46.5 \\
\hline Arp 220 & $\mathrm{~S} 2$ & 0.0181 & 470 & M97 & $150 \pm 4$ & SF-98 & 53.6 \\
\hline NGC 6104 & $\mathrm{~S} 1$ & 0.0279 & 500 & M97 & $148 \pm 35$ & NW95 & 39.5 \\
\hline NGC 6814 & $\mathrm{~S} 1$ & 0.0052 & 55 & M97 & $115 \pm 18$ & NW95 & 20.0 \\
\hline NGC 7172 & $\mathrm{~S} 2$ & 0.0086 & 580 & M97 & $180 \pm 40$ & Lon $+94 b$ & 55.1 \\
\hline Mrk 530 & $\mathrm{~S} 1$ & 0.0293 & 570 & M97 & $194 \pm 31$ & NW95 & 58.2 \\
\hline NGC 7674 & $\mathrm{~S} 2$ & 0.0295 & 195 & M97 & $144 \pm 32$ & NW95 & 31.3 \\
\hline NGC 7743 & $\mathrm{~S} 2$ & 0.0057 & 100 & M97 & $83 \pm 20$ & NW95 & 90.0 \\
\hline NGC 931 & S1 & 0.0164 & 500 & H89 & $151 \pm 32$ & NW95 & 81.8 \\
\hline M51 & $\mathrm{S} 2$ & 0.0016 & 130 & H89 & $82 \pm 11$ & NW95 & 30.0 \\
\hline NGC 5506 & $\mathrm{~S} 2$ & 0.0058 & 300 & H89 & $180 \pm 20$ & OOMM99 & 90.0 \\
\hline Mrk 273 & $\mathrm{~S} 2$ & 0.0373 & 450 & H89 & $160 \pm 60$ & $\mathrm{Jam}+99$ & 69.5 \\
\hline $3 \mathrm{C} 31$ & RG & 0.0169 & 450 & E05 & $254 \pm 22$ & SHI90 & 40.2 \\
\hline $3 \mathrm{C} 84$ & $\mathrm{RG}$ & 0.0176 & 200 & E05 & $272 \pm 61$ & NW95 & 57.9 \\
\hline $3 \mathrm{C} 120$ & RG & 0.0331 & 550 & E05 & $193 \pm 40$ & SHI90 & 62.2 \\
\hline 3C 293 & $\mathrm{RG}$ & 0.0448 & 700 & E05 & $185 \pm 20$ & $\mathrm{H}+85$ & 63.4 \\
\hline
\end{tabular}

Note. - a: Types of AGN. S1, S2 and RG represent Seyfert 1, Seyfert 2 and radio galaxy, respectively. b: Refereces for the CO line width data. M97: Maiolino et al. (1997); H89: Heckman et al. (1989); E05: Evans et al. (2005). c: References for the bulge velocity dispersion data. NW 95: Nelson \& Whittle (1995); OOKM95: Oliva et al. (1995); OOMM99: Oliva et al. 
(1999); TDT: Terlevich, Diaz \& Terlevich (1990); SWC93: Shaw, Wilkinson \& Carter (1993); HS98: Heraudean \& Simien (1998); SF-98: Shier \& Fisher (1998); Lon+94b: Longo et al. (1994); Jam+99: James et al. (1999); SHI90: Smith, Heckman \& Illingworth (1990); H+85: Heckmann et al. (1985). d: inclination data are from the Hyperleda database except for NGC 6814, whose inclination is taken from Whittle (1992). 